\title{
Multi-Objective Optimization of Two-Stage Helical Gear Train Using NSGA-II
}

\author{
R. C. Sanghvi, ${ }^{1}$ A. S. Vashi, ${ }^{2}$ H. P. Patolia, ${ }^{2}$ and R. G. Jivani ${ }^{2}$ \\ ${ }^{1}$ Department of Mathematics, G. H. Patel College of Engineering and Technology, Vallabh Vidyanagar 388120, India \\ ${ }^{2}$ Mechanical Engineering Department, B. V. Mahavidyalaya, Vallabh Vidyanagar 388120, India \\ Correspondence should be addressed to R. C. Sanghvi; rajeshsanghvi@gcet.ac.in
}

Received 31 May 2014; Revised 21 October 2014; Accepted 4 November 2014; Published 30 November 2014

Academic Editor: Liwei Zhang

Copyright (c) 2014 R. C. Sanghvi et al. This is an open access article distributed under the Creative Commons Attribution License, which permits unrestricted use, distribution, and reproduction in any medium, provided the original work is properly cited.

\begin{abstract}
Gears not only transmit the motion and power satisfactorily but also can do so with uniform motion. The design of gears requires an iterative approach to optimize the design parameters that take care of kinematics aspects as well as strength aspects. Moreover, the choice of materials available for gears is limited. Owing to the complex combinations of the above facts, manual design of gears is complicated and time consuming. In this paper, the volume and load carrying capacity are optimized. Three different methodologies (i) MATLAB optimization toolbox, (ii) genetic algorithm (GA), and (iii) multiobjective optimization (NSGA-II) technique are used to solve the problem. In the first two methods, volume is minimized in the first step and then the load carrying capacities of both shafts are calculated. In the third method, the problem is treated as a multiobjective problem. For the optimization purpose, face width, module, and number of teeth are taken as design variables. Constraints are imposed on bending strength, surface fatigue strength, and interference. It is apparent from the comparison of results that the result obtained by NSGA-II is more superior than the results obtained by other methods in terms of both objectives.
\end{abstract}

\section{Introduction}

Designing a new product consists of several parameters and phases, which differ according to the depth of design, input data, design strategy, procedures, and results. Mechanical design includes an optimization process in which designers always consider certain objectives such as strength, deflection, weight, wear, and corrosion depending on the requirements. However, design optimization for a complete mechanical assembly leads to a complicated objective function with a large number of design variables. So it is a better practice to apply optimization techniques for individual components or intermediate assemblies than a complete assembly. For example, in an automobile power transmission system, optimization of gearbox is computationally and mathematically simpler than the optimization of complete system. The preliminary design optimization of two-stage helical gear train has been a subject of considerable interest, since many high-performance power transmission applications require high-performance gear train.
A traditional gear design involves computations based on tooth bending strength, tooth surface durability, tooth surface fatigue, interference, efficiency, and so forth. Gear design involves empirical formulas, different graphs and tables, which lead to a complicated design. Manual design is very difficult considering the above facts and there is a need for the computer aided design of gears. With the aid of computer, design can be carried out iteratively and the design variables which satisfy the given conditions can be determined. The design so obtained may not be the optimum one, because in the above process the design variables so obtained satisfy only one condition at a time; for example, if module is calculated based on bending strength, the same module is substituted to calculate the surface durability. It is accepted if it is within the strength limit of surface durability; otherwise it is changed accordingly. So optimization methods are required to determine design variables which simultaneously satisfy the given conditions. As the optimization problem involves the objective function and constraints that are not stated as explicit functions of the design variables, it is hard to solve 
it by classical optimization methods. Moreover, increasing demand for compact, efficient, and reliable gears forces the designer to use optimal design methodology.

Huang et al. [1] developed interactive physical programming approach of the optimization model of three-stage spur gear reduction unit with minimum volume, maximum surface fatigue life, and maximum load-carrying capacity as design objectives and core hardness, module, face width of gear, tooth numbers of pinion, tooth numbers of gear, and diameter of shaft as design variables. In this modeling, tooth bending fatigue failure, shaft torsional stress, face width, interference, and tooth number are considered as constraints. The MATLAB constrained optimization package is used to solve this nonlinear programming problem. Jhalani and Chaudhary [2] discussed the various parameters which can affect the design of the gearbox for knee mounted energy harvester device and later it frames the optimization problem of mass function based on the dimensions of gearbox for the problem. The problem is solved using multistart approach of MATLAB global optimization toolbox and value of global optimum function is obtained considering all the local optimum solutions of problem. Tong and Walton [3] also selected center distance and volume as objectives for the internal gears. Numbers of teeth of gear and pinion and modules are considered as variables for the optimization and "belt zone search" and "half section algorithm" are applied as optimization methods. Savsani et al. [4] presented the application of two advanced optimization algorithms known as particle swarm optimization (PSO) and simulated annealing (SA) to find the optimal combination of design parameters for minimum weight of a spur gear train. Wei et al. [5] developed a mathematical model of optimization considering the basic design parameters, mainly tooth number, modulus, face width, and helix angle of gearbox as design variables and reduction of weight or volume as an objective. The model is illustrated by an example of the gearbox of medium-sized motor truck. Optimization tool box of MATLAB and sequential quadratic programming (SQP) method were used to optimize the gearbox. The design criterion and performance conditions of gearbox are treated as constraints.

Mendi et al. [6] studied the dimensional optimization of motion and force transmitting components of a gearbox by GA. It is aimed at obtaining the optimal dimensions for gearbox shaft, gear, and the optimal rolling bearing to minimize the volume which can carry the system load using GA. The results obtained by GA optimization are compared to those obtained by analytical methods. Mogal and Wakchaure [7] used GA as evolutionary techniques for optimization of worm and worm wheel. The main objective for optimization is minimizing the volume; here other objectives are considered as constraints. Gear ratio, face width, and pitch circle diameter of worm and worm wheel are the design variables for objectives. Constraints are center distance, deflection of worm and beam strength of worm gear. Yokota et al. [8] formulated an optimal weight design problem of a gear for a constrained bending strength of gear, torsional strength of shafts, and each gear dimension as a nonlinear integer programming (NIP) problem and solved it directly by using an improved GA. The efficiency of the proposed method is confirmed by showing the improvement in weight of gears and space area. Buiga and Popa [9] presented an optimal design mass minimization problem of a single-stage helical gear unit, complete with the sizing of shafts, gearing, and housing using GAs. Mohan and Seshaiah [10] discussed the optimization of spur gear set for its center distance, weight and tooth deflections with module, face width, and number of teeth on pinion as decision variables subject to constraints on bending stress and contact stress. Three materials, namely, Cast Iron, C-45, and Alloy Steel (15Ni2 Cr1), are considered. The gear parameters obtained from GA are compared with the conventional results.

Thompson et al. [11] presented a generalized optimal design formulation with multiple objectives which is, in principle, applicable to a gear train of arbitrary complexity. The methodology is applied to the design of two-stage and threestage spur gear reduction units, subject to identical loading conditions and other design criteria. The approach serves to extend traditional design procedures by demonstrating the tradeoff between surface fatigue life and minimum volume using a basic multiobjective optimization procedure. Padmanabhan et al. [12] investigated that in many real-life problems, objectives under consideration conflict with each other, and optimizing a particular solution with respect to a single objective can result in unacceptable results with respect to the other objectives. Multiobjective formulations are realistic models for many complex engineering optimization problems. Ant Colony Optimization was developed specifically for a worm gear drive problem with multiple objectives. Deb and Jain [13] demonstrated the use of a multiobjective evolutionary algorithm, namely, Nondominated Sorting Genetic Algorithm (NSGA-II), which is capable of solving the original problem involving mixed discrete and real-valued parameters and more than one objective.

In this paper, two stages of helical gear train are considered. There are several factors, which affect the assembly as well as working condition. They are not generally considered in literature. The optimization model formulated here includes these factors in constraints. A GUI is developed which facilitates the input of various combinations of input data. Moreover, a code of GA is also developed. The optimization is carried out using optimization toolbox of MATLAB and GA and the results obtained by both of the methods are compared. These methods are applied to minimize the volume only. The resulting values of the parameters are applied to find the maximum load carrying capacity. In true sense, the problem is solved as two single objective problems, one at a time. Moreover, NSGA-II is applied to the problem to solve it as a multiobjective problem.

\section{Formulation of Problem}

The optimization model of two-stage helical gear reduction unit is formulated in this section, with minimum volume and maximum load carrying capacity as design objectives. The schematic illustration of two-stage helical gear reduction unit is shown in Figure 1. As it is a case of two-stage gear reduction, 


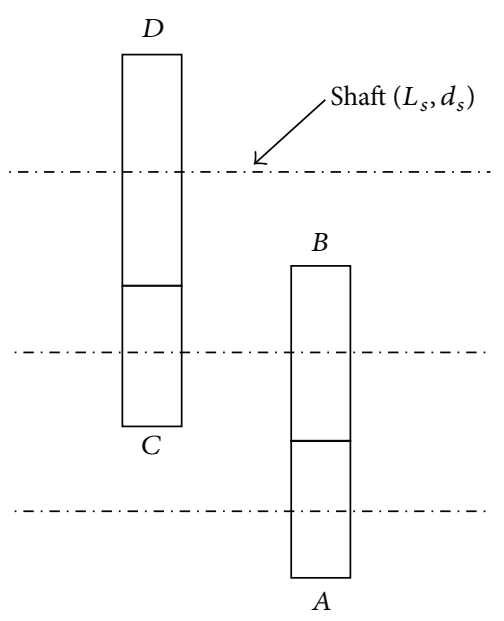

FIGURE 1: Schematic illustration of two-stage helical gear train.

the gear ratios between first pair and second pair are chosen in such a way that their values are feasible and their product remains the same as that of required.

2.1. Design Variables. The mainly affected parameters of gear from the volume point of view are face width, module, and number of teeth of gear. These parameters directly or indirectly affect the objectives widely. So, the design vector $X$ is

$$
X=\left\{b_{A}, b_{C}, m_{n A}, m_{n C}, z_{A}, z_{B}, z_{C}, z_{D}\right\}
$$

where $z_{A}, z_{B}, z_{C}$, and $z_{D}$ are the number of teeth of gears $A, B$, $C$, and $D$, respectively; $b_{A}$ and $b_{C}$ are the face widths of gears $A$ and $C$, respectively; $m_{n A}$ and $m_{n C}$ are the normal modules of gears $A$ and $C$, respectively. Here it is assumed that all gears are of the same material (say with the same Brinell hardness number) and are of the same helix angle.

2.2. Objective Functions. For the optimization, first the volume of the two-stage helical gear train is minimized. After achieving the optimal value of design variables for minimum volume, those values of variables are applied to maximize the load carrying capacity of both of the stages. From both of these stages, the minimum load carrying capacity out of the two is chosen as the maximum capacity for the gear train.

The optimization model of two-stage helical gear trains is derived as follows.

Considering the dimensions of the three shafts constant, the volume of the gear train is

$$
\begin{gathered}
V=\frac{\pi}{4}\left[\left(d_{A}{ }^{2}+d_{B}{ }^{2}\right) b_{A}+\left(d_{C}{ }^{2}+d_{D}{ }^{2}\right) b_{C}\right. \\
\left.+d_{1}{ }^{2} L_{1}+d_{2}{ }^{2} L_{2}+d_{3}{ }^{2} L_{3}\right]
\end{gathered}
$$

and the load carrying capacity $P$ is given as [14]

$$
P_{\mathrm{eff}}=C_{s} P_{t}+P_{d}
$$

Referring to $P_{\text {eff }}$ of the two stages as $P_{1}$ and $P_{2}$ further can be written as

$$
\begin{aligned}
P_{1}= & \frac{2 C_{S} M_{t 1}}{d_{A}} \\
& +\frac{21 \pi n_{1}\left(C e_{1} b_{A} d_{A}{ }^{1.5} \cos ^{2} \psi+2 d_{A}{ }^{0.5} M_{t 1}\right) \cos \psi}{21 \pi d_{A}{ }^{1.5} n_{1}+60,000 \sqrt{C e_{1} b_{A} d_{A} \cos ^{2} \psi+2 M_{t 1}}}, \\
P_{2}= & \frac{2 C_{S} M_{t 2}}{d_{C}} \\
& +\frac{21 \pi n_{2}\left(C e_{2} b_{C} d_{C}^{1.5} \cos ^{2} \psi+2 d_{C}^{0.5} M_{t 2}\right) \cos \psi}{21 \pi d_{C}^{1.5} n_{2}+60,000 \sqrt{C_{2} b_{C} d_{C} \cos ^{2} \psi+2 M_{t 2}}},
\end{aligned}
$$

where $d_{1}, d_{2}, d_{3}$ and $L_{1}, L_{2}, L_{3}$ represent the diameters of shaft and lengths of shaft $1,2,3$, respectively. The factors $C_{S}$ and $C$ denote service factor and deformation factor, respectively. $M_{t i}$ is the transmitted torque and $e_{A}$ and $e_{C}$ are sum of error between first meshing teeth and second meshing teeth, respectively.

Thus the objectives can be written for minimum volume and maximum load carrying capacity as

$$
V_{\min }, P_{\max }=\left\{P_{1}, P_{2}\right\} \text {. }
$$

2.3. Constraints. When the gear tooth is considered as a cantilever beam, the bending strength in working condition should not exceed standard endurance limit, $S_{n}$. From Lewis equation, the constraint on bending strength is

$$
\frac{F_{t} P}{b Y} \leq S_{n}
$$

where $F_{t}=\left(k W \times 10^{3}\right) / v, v=\pi d n /\left(60 \times 10^{3}\right), P$ is diametral pitch, $b$ is face width, and $Y$ is Lewis factor.

However, in this work, the factors affecting bending strength during the production and assembly, such as velocity factor, overload factor, and mounting factor to name a few, are not taken into consideration. So, after adding the effects of these factors, the new constraints on bending strength for both of the gear pairs can be expressed [15] as

$$
\begin{aligned}
& \frac{F_{t A} P_{A}}{b_{A} J_{A}} K_{v A} K_{o}\left(0.93 K_{m A}\right)-S_{n}^{\prime} C_{L} C_{G A} C_{S} k_{r} k_{t} k_{\mathrm{ms}} \leq 0, \\
& \frac{F_{t C} P_{C}}{b_{C} J_{C}} K_{v C} K_{o}\left(0.93 K_{m C}\right)-S_{n}^{\prime} C_{L} C_{G C} C_{S} k_{r} k_{t} k_{\mathrm{ms}} \leq 0,
\end{aligned}
$$

where $J$ is geometry factor which includes the Lewis form factor $Y$ and a stress concentration factor. $K_{v}, K_{o}$, and $K_{m}$ denote velocity or dynamic factor, overload factor, and mounting factor, respectively. $S_{n}^{\prime}$ is standard R. R. Moore endurance limit. $C_{L}, C_{G}$, and $C_{S}$ denote load factor, gradient factor, and service factor, respectively. $k_{t}, k_{r}$, and $k_{\mathrm{ms}}$ denote temperature factor, reliability factor, and mean stress factor, respectively.

Gear teeth are vulnerable to various types of surface damage. As was the case with rolling-element bearings, 
gear teeth are subjected to Hertz contact stresses, and the lubrication is often elastohydrodynamic. Excessive loading and lubrication breakdown can cause various combinations of abrasion, pitting, and scoring. It will become evident that gear-tooth surface durability is a more complex matter than the capacity to withstand gear-tooth-bending fatigue.

After including all the parameters the surface fatigue constraint formula can be written [15] as

$$
\begin{aligned}
& C_{p} \sqrt{\frac{F_{t A}}{b_{A} d_{A} I_{A}} \times \frac{\cos \psi}{0.95 \mathrm{CR}_{A}} \times K_{v A} K_{o}\left(0.93 K_{m A}\right)} \\
& -S_{f_{c} C_{\mathrm{Li}} C_{R} \leq 0,} \\
& d C_{p} \sqrt{\frac{F_{t C}}{b_{C} d_{C} I_{C}} \times \frac{\cos \psi}{0.95 \mathrm{CR}_{C}} \times K_{v C} K_{o}\left(0.93 K_{m C}\right)} \\
& -S_{f_{c} C_{\mathrm{Li}} C_{R} \leq 0,}
\end{aligned}
$$

where $C_{p}, C_{\mathrm{Li}}$, and $C_{R}$ denote elastic coefficient factor, life factor, and reliability factor, respectively. $I_{A}$ and $I_{C}$ are dimensionless constants and $\mathrm{CR}_{A}$ and $\mathrm{CR}_{C}$ are contact ratios. $S_{f c}$ represents surface fatigue strength.

While designing the gear, interference is the main factor to consider. Interference usually takes place in the gear. So formulation of the optimization problem must take care of interference. To remove interference, the following constraints should be satisfied (see $[15,16])$ :

$$
\begin{gathered}
r_{a A}-\sqrt{r_{b A}^{2}+c_{A}^{2} \sin ^{2} \phi} \leq 0, \\
r_{a C}-\sqrt{r_{b C}^{2}+c_{C}^{2} \sin ^{2} \phi} \leq 0, \\
\frac{2}{\sin ^{2} \phi}-z_{A} \leq 0, \\
\frac{2}{\sin ^{2} \phi}-z_{B} \leq 0, \\
\frac{2}{\sin ^{2} \phi}-z_{C} \leq 0, \\
\frac{2}{\sin ^{2} \phi}-z_{D} \leq 0 .
\end{gathered}
$$

\section{Methods of Solution}

Since there are many input parameters such as dimensions of shafts, gear train parameters, material properties, working condition of gear train, and factor affecting production and assembly, a GUI is prepared as shown in Figures 2, 3, 4, and 5. The problem is solved by following three ways:

(i) using optimization toolbox of MATLAB,

(ii) using code developed for GA,

(iii) using multiobjective optimization (NSGA-II) technique.

The ranges of the problem variables are taken as reference from manufacturer's catalog [17] and these ranges for $b_{A}, m_{A}$,

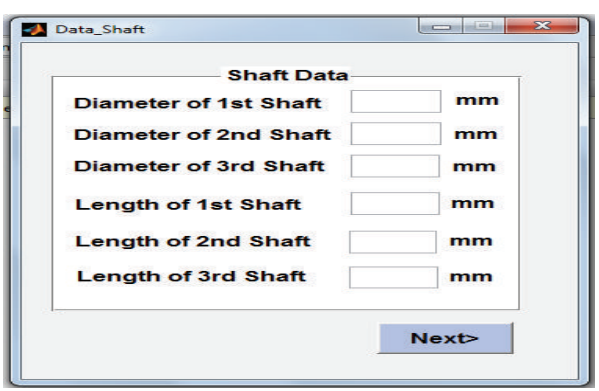

FIGURE 2: Input data through "Data_Shaft."

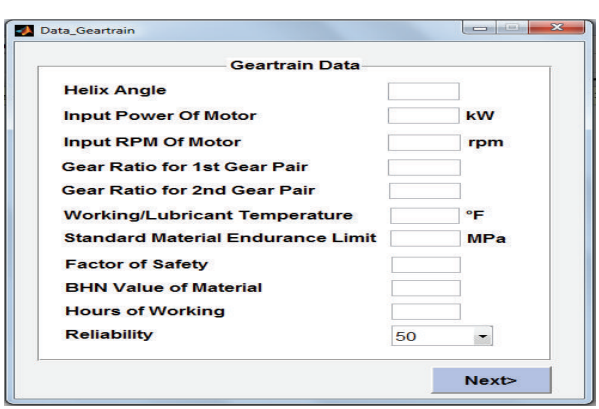

Figure 3: Input data through "Data_Geartrain.”

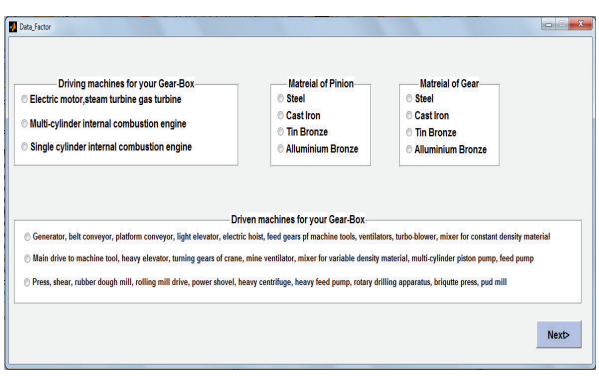

FIGURE 4: Input data through "Data_Factor."

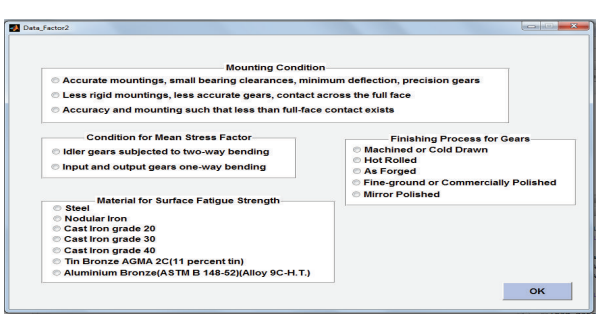

FIGURE 5: Input data through “Data_Factor2.”

$z_{A}, z_{B}, b_{C}, m_{C}, z_{C}$, and $z_{D}$ are taken as 60-80, 4-12, 14-20, $44-65,85-105,3-10,14-20$, and 77-110, respectively.

3.1. Using the Optimization Toolbox of MATLAB. In this method, first the volume of the gear train is minimized. The resulting values of the parameters are used to determine the load carrying capacities of both of the shafts. The minimum of them is considered as the maximum load carrying capacity. In this way, a multiobjective problem is reduced to a single 
objective problem. The "optimtool" feature of MATLAB is useful for different kinds of optimization problem. In the problem discussed here, constraints are nonlinear. So "fmincon" function of MATLAB applicable for nonlinear constraint minimization is used for the optimization. There are different algorithms and methods available under this option in the optimization toolbox. Interior-point algorithm is chosen among them as it handles large, sparse problems, as well as small dense problems. Moreover, the algorithm satisfies bounds at all iterations and can recover from $\mathrm{NaN}$ or Inf results. It is a large-scale algorithm widely used for this type of problems.

This function requires a point to start with, the choice of which is arbitrary. The results obtained for face width of gear $A$, module of gear $A$ (and $B$ ), number of teeth of gear $A$, number of teeth of gear $B$, face width of gear $C$, module of gear $C$ (and $D$ ), number of teeth of gear $C$, and number of teeth of gear $D$ are $60,4,17.097,53.737,85,3,17.097$, and 94.035 , respectively. The corresponding volume is $1.948 \times$ $10^{7} \mathrm{~mm}^{3}$. The result remains invariant if other starting points are chosen. For the value of load carrying capacity, the values for first and second stages are $3.3352 \times 10^{4} \mathrm{~N}$ and $3.3909 \times$ $10^{4} \mathrm{~N}$. So, from these values, the load carrying capacity of the gear train is selected as $3.3352 \times 10^{4} \mathrm{~N}$.

3.2. Optimization Using Genetic Algorithm. The same strategy used in the first method is also applied here to deal with a multiobjective problem. First the volume is minimized and then minimum of the resulting two load carrying capacities is chosen as the maximum load carrying capacity. The only difference is that to minimize the volume, GA is used. As discussed in introduction, many designs are characterized by mixed continuous-discrete variables and discontinuous and nonconvex design spaces. Standard nonlinear programming techniques are not capable of solving these types of problems. They usually find relative optimum that is closest to the starting point. GA is well suited for solving such problems, and in most cases, they can find the global optimum solution with high probability. Actually the idea of evolutionary computing was introduced in the 1960s by I. Rechenberg in his work "Evolution strategies" which was then developed by others. GAs were invented and developed by Holland [18]. The basic ideas of analysis and design based on the concepts of biological evolution can be found in the work of Rechenberg [19]. Philosophically, GAs are based on Darwin's theory of survival of the fittest and also are based on the principles of natural genetics and natural selection. The basic elements of natural genetics-reproduction, cross-over, and mutation are used in the genetic search procedures.

GA is a search algorithm based on the conjecture of natural selection and genetics. The features of GA are different from the other search techniques in several aspects as follows:

(i) the algorithm is a multipath that searches many peaks in parallel, hence reducing the possibility of local minimum trapping;

(ii) GAs work with coding of the parameter set, not the parameters themselves; (iii) GAs evaluate a population of points, not a single point;

(iv) GAs use objective function information, not derivations or other auxiliary knowledge, to determine the fitness of the solution;

(v) GAs use probabilistic transition rules, not deterministic rules in the generation of the new population.

3.2.1. Outline of Basic Genetic Algorithm. The basic procedure of GA as outlined in [20] is as follows:

(1) [Start] Generate random population of $n$ chromosomes (suitable solution for problem)

(2) [Fitness] Evaluate the fitness $f(x)$ of each chromosome $x$ in the population

(3) [New population] Create a new population by repeating following steps until the new population is complete:

(i) [Selection] Select two parent chromosomes from a population according to their fitness (the better fitness, the bigger chance to be selected.)

(ii) [Crossover] With a crossover probability, $P_{C}$ crossover the two parents to from two new offspring. If no crossover was performed, offspring is the exact copy of parents.

(iii) [Mutation] With a mutation probability, $P_{m}$ mutate new offspring at each locus (position in chromosome.)

(iv) [Accepting] Place new offspring in the new population.

(4) [Replace] Use new generated population for a further run of the algorithm.

(5) [Test] If the end condition is satisfied, stop and return the best solution in current population.

(6) [Loop] Go to Step (2).

3.2.2. Implementation of Genetic Algorithm. Extensive experiments are carried out for different combinations of population size and number of generations. It is observed that the results remain consistent when the population size is 90 and number of generations is 90 . So, eleven good results with this population size and number of generations are shown in Table 1 in which the 10th solution is the best. Corresponding load carrying capacities of the first and the second pair are $32.86 \mathrm{kN}$ and $34.16 \mathrm{kN}$, respectively. So, the load carrying capacity of gear train is selected as $32.86 \mathrm{kN}$ for which optimum volume is $2.0396 \times 10^{7} \mathrm{~mm}^{3}$.

3.3. Optimization Using NSGA-II. In this case, the problem is considered as a multiobjective problem. So, both objectives are treated together. In general, in case of multiobjective optimization, the objectives are conflicting. So, a single solution cannot be accepted as the best solution. Instead, a set of solutions is obtained which are better than the 
TABLE 1: Results of GA for population size of 90 and 90 generations.

\begin{tabular}{lccccccccc}
\hline Sr. number & $\begin{array}{c}b_{A} \\
(\mathrm{~mm})\end{array}$ & $\begin{array}{c}m_{A} \\
(\mathrm{~mm})\end{array}$ & $z_{A}$ & $z_{B}$ & $\begin{array}{c}b_{C} \\
(\mathrm{~mm})\end{array}$ & $\begin{array}{c}m_{C} \\
(\mathrm{~mm})\end{array}$ & $z_{C}$ & $z_{D}$ & $V$ Volume $\left(10^{7} \times \mathrm{mm}^{3}\right)$ \\
\hline 1 & 67.06 & 4 & 18 & 57 & 90.18 & 3 & 18 & 99 & 2.166 \\
2 & 64.07 & 4 & 19 & 60 & 85.29 & 3 & 18 & 99 & 2.088 \\
3 & 62.07 & 4 & 18 & 57 & 86.7 & 3 & 18 & 99 & 2.058 \\
4 & 75.1 & 4 & 18 & 57 & 85.91 & 3 & 18 & 99 & 2.111 \\
5 & 66.07 & 4 & 20 & 63 & 85.23 & 3 & 18 & 99 & 2.132 \\
6 & 63.7 & 4 & 19 & 60 & 87.74 & 3 & 18 & 99 & 2.104 \\
7 & 62 & 4 & 18 & 57 & 86.82 & 3 & 18 & 99 & 2.059 \\
8 & 60.71 & 4 & 18 & 57 & 86.04 & 3 & 18 & 99 & 2.047 \\
9 & 61.77 & 4 & 18 & 57 & 86.47 & 3 & 18 & 99 & 99 \\
10 & 60.01 & 4 & 18 & 57 & 85.31 & 3 & 18 & 99 \\
11 & 62.85 & 4 & 19 & 60 & 85.31 & 3 & 19 & 99 \\
\hline
\end{tabular}

TABLE 2: Results of NSGA-II for population size of 500 and 500 generations.

\begin{tabular}{lcccccccccc}
\hline Sr. number & $\begin{array}{c}b_{A} \\
(\mathrm{~mm})\end{array}$ & $\begin{array}{c}m_{A} \\
(\mathrm{~mm})\end{array}$ & $z_{A}$ & $z_{B}$ & $\begin{array}{c}b_{C} \\
(\mathrm{~mm})\end{array}$ & $\begin{array}{c}m_{C} \\
(\mathrm{~mm})\end{array}$ & $z_{C}$ & $z_{D}$ & $\begin{array}{c}\text { Volume } \\
\left(10^{7} \times \mathrm{mm}^{3}\right)\end{array}$ & Load carrying capacity $(\mathrm{kN})$ \\
\hline 1 & 80.00 & 4 & 18 & 57 & 87.62 & 3 & 18 & 99 & 2.048 & 35.306 \\
2 & 60.39 & 4 & 18 & 57 & 85.00 & 3 & 18 & 99 & 1.951 & 33.718 \\
3 & 79.04 & 4 & 18 & 57 & 90.83 & 3 & 18 & 99 & 2.066 & 35.837 \\
4 & 80.00 & 4 & 18 & 57 & 90.98 & 3 & 18 & 99 & 2.070 & 35.941 \\
5 & 71.48 & 4 & 18 & 57 & 87.62 & 3 & 18 & 99 & 2.013 & 35.996 \\
6 & 74.77 & 4 & 18 & 57 & 87.89 & 3 & 18 & 99 & 2.027 & 34.383 \\
7 & 66.80 & 4 & 18 & 57 & 86.33 & 3 & 18 & 99 & 1.993 & 34.076 \\
8 & 63.45 & 4 & 18 & 57 & 85.00 & 3 & 18 & 99 & 1.963 & \\
\hline
\end{tabular}

other solutions in terms of both objectives which are called Pareto optimal solutions. Since evolutionary algorithms are population based, they are the natural choice for solving this kind of problem. In NSGA-II, the iterative procedure starts from an arbitrary population of solutions and gradually the algorithm converges to a population of solutions lying on the Pareto optimal front with higher diversity. The operators applied are the same as those of GA, namely, selection, crossover, and mutation. The tournament selection operator is applied which also takes care of constraints. However, in case of multiobjective optimization, additional task is to obtain solutions which are as diverse as possible. For that, the sharing function approach is used. Crossover and mutation operators are applied as usual. A detailed discussion of this algorithm is found in [21]. The standard code available at [22] is modified according to authors' need.

As a result of NSGA-II, out of the population size of 50 and number of generations of 500, eight better results are selected and shown in Table 2. It has been observed that fulfilling both of the objectives together, the second last solution is the compromised one. Corresponding optimum volume and load carrying capacity of the train are $1.993 \times$ $10^{7} \mathrm{~mm}^{3}$ and $34.38 \mathrm{kN}$, respectively.

\section{Results and Discussion}

There are several comments in order. The number of teeth of gear $A$ and gear $C$ in the manufacturer's design is 14 . It creates interference in working condition. To eliminate it, manufacturer produces stub tooth instead of normal tooth which is not advisable. The introduction of the constraints on interference in the proposed formulation takes care of this problem as the number of teeth of gear $A$ and gear $C$ will definitely exceed 17 . The major problem with the inbuilt "fmincon" function of MATLAB is that it considers all the variables real. As a result, one has to round the optimum value of integer variable to the nearest integer. So, the optimum value of number of teeth of gear $A$ and gear $C$ is rounded off to 18. To maintain the gear ratios, the numbers of teeth of gear $B$ and gear $D$ have to be selected as 56 and 100 , respectively, which are quite far from their actual optimum values obtained using toolbox.

However, GA can deal with both types of variables, integer and real, very easily by choosing appropriate string length. But in this case also, numbers of teeth of gear $B$ and gear $D$ have to be changed to 58 and 100, respectively, because of manufacturing inconveniences. NSGA-II selects 57 and 99 as the numbers of teeth of gear $B$ and gear $D$ which is better than both of the above results. The results are presented in Table 3 .

\section{Conclusion and Future Scope}

Result comparison table shows that in the first two cases minimization of volume took place while load carrying 
TABLE 3: Comparison of results.

\begin{tabular}{|c|c|c|c|c|}
\hline Variables and objectives & Catalog value & $\begin{array}{c}\text { Optimization toolbox } \\
\text { value } \\
\text { (round off) }\end{array}$ & GA (round off) & NSGA-II (round off) \\
\hline Face width of gear $A(\mathrm{~mm})$ & 70 & 60 & 60 & 67 \\
\hline Module of gear $A(\mathrm{~mm})$ & 7 & 4 & 4 & 4 \\
\hline Number of teeth of gear $A$ & 14 & 18 & 18 & 18 \\
\hline Number of teeth of gear $B$ & 44 & 56 & 58 & 57 \\
\hline Face width of gear $C(\mathrm{~mm})$ & 95 & 85 & 85 & 86 \\
\hline Module of gear $C(\mathrm{~mm})$ & 3.5 & 3 & 3 & 3 \\
\hline Number of teeth of gear $C$ & 14 & 18 & 18 & 18 \\
\hline Number of teeth of gear $D$ & 77 & 100 & 100 & 99 \\
\hline Volume $\left(\mathrm{mm}^{3}\right)$ & $2.293 \times 10^{7}$ & $2.0408 \times 10^{7}$ & $2.0602 \times 10^{7}$ & $1.993 \times 10^{7}$ \\
\hline Load carrying capacity $(\mathrm{N})$ & $3.405 \times 10^{4}$ & $3.2861 \times 10^{4}$ & $3.2874 \times 10^{4}$ & $3.4383 \times 10^{4}$ \\
\hline
\end{tabular}

capacity is reduced marginally low. While using optimization toolbox, volume is reduced by $15.04 \%$ but when their nearer integer value of variable is selected because of inconveniences in manufacturing, volume is reduced by $10.9 \%$. For the GA the volume is reduced by $11.05 \%$ but when their nearer integer value of variable is selected, volume is reduced by $10.15 \%$. Though these results show that optimization tool box gives better result than GA, it is better to use GA for global optimum value as optimization toolbox which gives results closest to the starting point and GA finds the more convenient solution with high probability of manufacturing. However, NSGA-II gives the best result compared to both of the above methods as it is superior in terms of both of the objectives, minimum volume and maximum load carrying capacity. For the NSGA-II, the volume is reduced by $13.08 \%$ and load carrying capacity is increased by $1 \%$.

The problem can be extended to more than two stages. Other recently developed evolutionary algorithms such as PSO and cuckoo search can also be tried to solve this problem. Similar approach can be followed in case of other applications, such as minimization of weight of spring and minimization of weight of pulley system.

\section{Conflict of Interests}

The authors declare that there is no conflict of interests regarding the publication of this paper.

\section{References}

[1] H.-Z. Huang, Z.-G. Tian, and M. J. Zuo, "Multiobjective optimization of three-stage spur gear reduction units using interactive physical programming," Journal of Mechanical Science and Technology, vol. 19, no. 5, pp. 1080-1086, 2005.

[2] D. Jhalani and H. Chaudhary, "Optimal design of gearbox for application in knee mounted biomechanical energy harvester," International Journal of Scientific \& Engineering Research, vol. 3, no. 10, pp. 1071-1075, 2012.

[3] B. S. Tong and D. Walton, "The optimisation of internal gears," International Journal of Machine Tools and Manufacture, vol. 27, no. 4, pp. 491-504, 1987.
[4] V. Savsani, R. V. Rao, and D. P. Vakharia, "Optimal weight design of a gear train using particle swarm optimization and simulated annealing algorithms," Mechanism and Machine Theory, vol. 45, no. 3, pp. 531-541, 2010.

[5] H. Wei, F. Lingling, L. Xiohuai, W. Zongyian, and Z. Leisheng, "The structural optimization of gearbox based on sequential quadratic programming method," in Proceedings of the 2nd International Conference on Intelligent Computing Technology and Automation (ICICTA '09), pp. 356-359, Hunan, China, October 2009.

[6] F. Mendi, T. Başkal, K. Boran, and F. E. Boran, "Optimization of module, shaft diameter and rolling bearing for spur gear through genetic algorithm," Expert Systems with Applications, vol. 37, no. 12, pp. 8058-8064, 2010.

[7] Y. K. Mogal and V. D. Wakchaure, "A multi-objective optimization approach for design of worm and worm wheel based on genetic algorithm," Bonfring International Journal of Man Machine Interface, vol. 3, pp. 8-12, 2013.

[8] T. Yokota, T. Taguchi, and M. Gen, "A solution method for optimal weight design problem of the gear using genetic algorithms," Computers \& Industrial Engineering, vol. 35, no. 34, pp. 523-526, 1998.

[9] O. Buiga and C.-O. Popa, "Optimal mass design of a single-stage helical gear unit with genetic algorithms," Proceedings of the Romanian Academy Series A-Mathematics Physics Technical Sciences Information Science, vol. 13, no. 3, pp. 243-250, 2012.

[10] Y. Mohan and T. Seshaiah, "Spur gear optimization by using genetic algorithm," International Journal of Engineering Research and Applications, vol. 2, pp. 311-318, 2012.

[11] D. F. Thompson, S. Gupta, and A. Shukla, "Tradeoff analysis in minimum volume design of multi-stage spur gear reduction units," Mechanism and Machine Theory, vol. 35, no. 5, pp. 609627, 2000.

[12] S. Padmanabhan, M. Chandrasekaran, and V. Srinivasa, "Design optimization of worm Gear drive," International Journal of Mining, Metallurgy and Mechanical Engineering, vol. 1, pp. 57-61, 2013.

[13] K. Deb and S. Jain, "Multi-speed gearbox design using multi-objective evolutionary algorithms," Journal of Mechanical Design, Transactions of the ASME, vol. 125, no. 3, pp. 609-619, 2003.

[14] V. B. Bhandari, Design of Machine Elements, Tata McGraw-Hill, 2010. 
[15] R. C. Juvinall and K. M. Marshek, Fundamentals of Machine Component Design, John Wiley \& Sons, 2011.

[16] G. Maitra, Handbook of Gear Design, Tata McGraw-Hill, 2nd edition, 2003.

[17] Design Catalog of Hi-Tech Drive Pvt. Ltd. Plot No. 443/A, GIDC, V. U. Nagar, Gujarat, India.

[18] J. H. Holland, Adaptation in Natural and Artificial Systems, University of Michigan Press, Ann Arbor, Mich, USA, 1975.

[19] I. Rechenberg, Cybernetic Solution Path of an Experimental Problem, Library Translation 1122, Royal Aircraft Establishment, Farnborough, Hampshire, UK, 1965.

[20] P. E. Amiolemhen and A. O. A. Ibhadode, "Application of genetic algorithms - determination of the optimal machining parameters in the conversion of a cylindrical bar stock into a continuous finished profile," International Journal of Machine Tools and Manufacture, vol. 44, no. 12-13, pp. 1403-1412, 2004.

[21] K. Deb, Multi-Objective Optimization Using Evolutionary Algorithms, John Wiley \& Sons, New York, NY, USA, 2009.

[22] http://www.mathworks.in/matlabcentral/fileexchange/31166ngpm-a-nsga-ii-program-in-matlab-v1-4. 


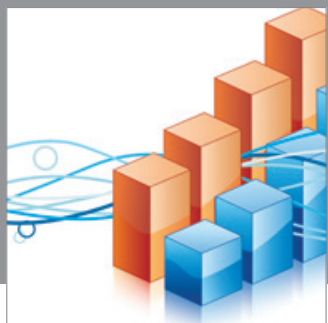

Advances in

Operations Research

mansans

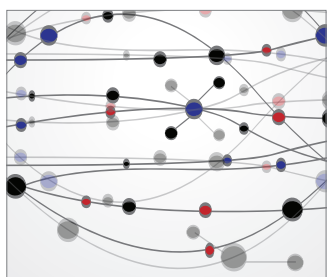

The Scientific World Journal
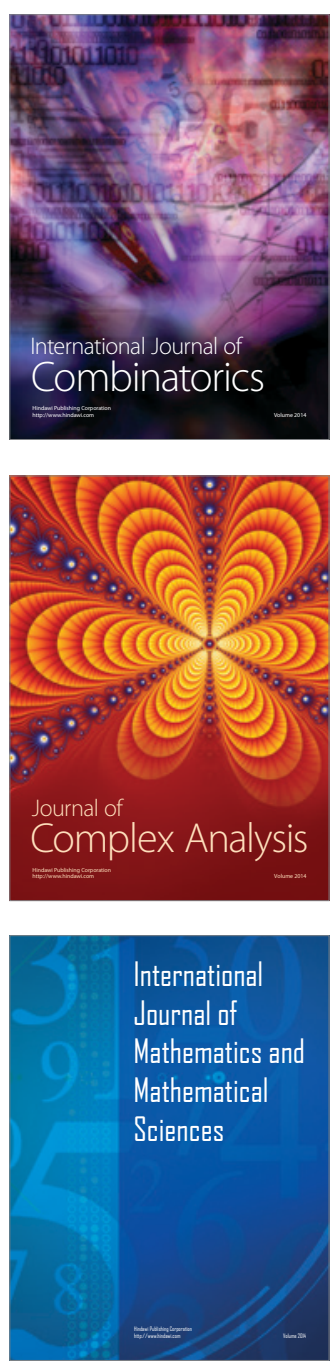
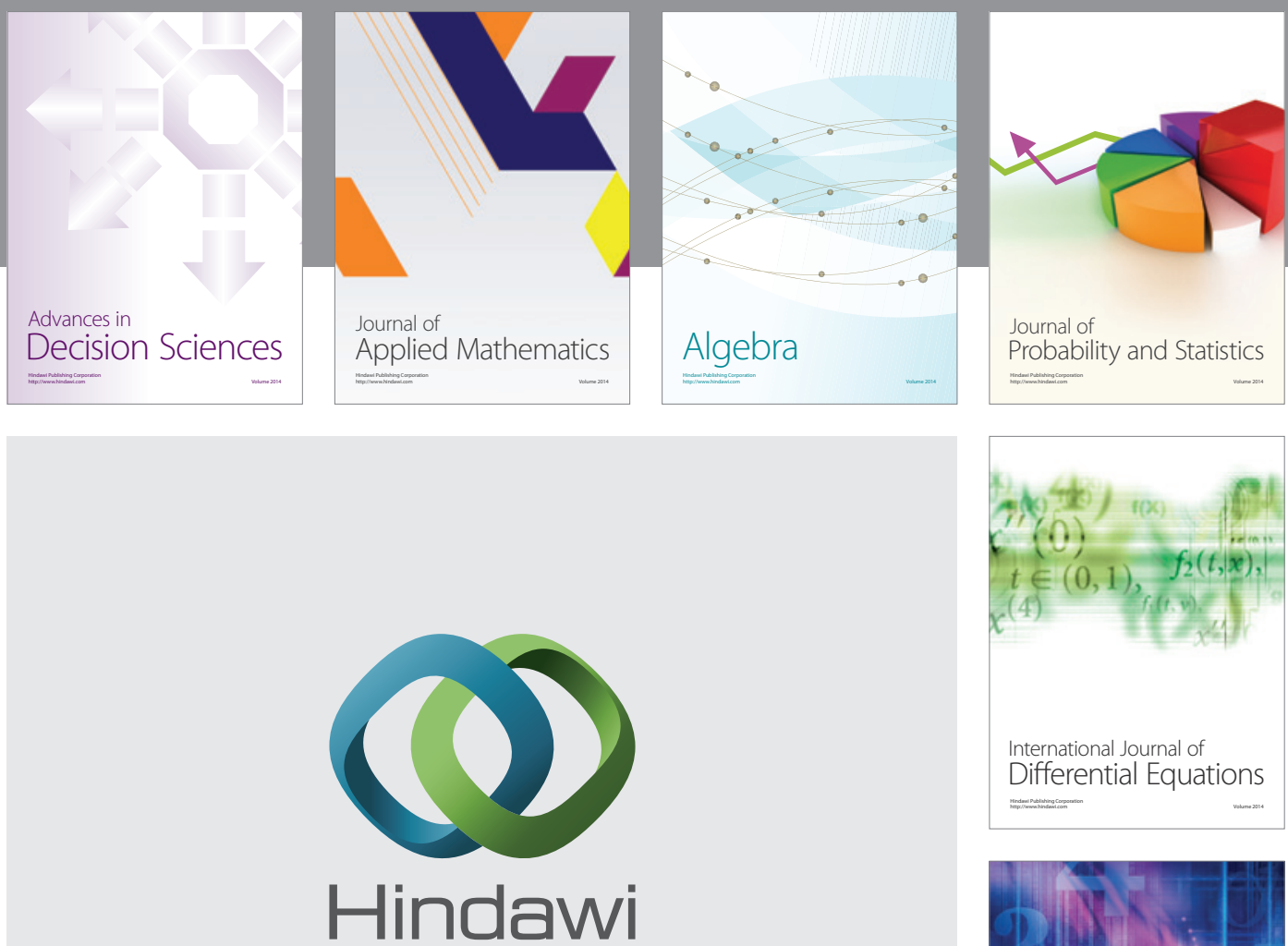

Submit your manuscripts at http://www.hindawi.com
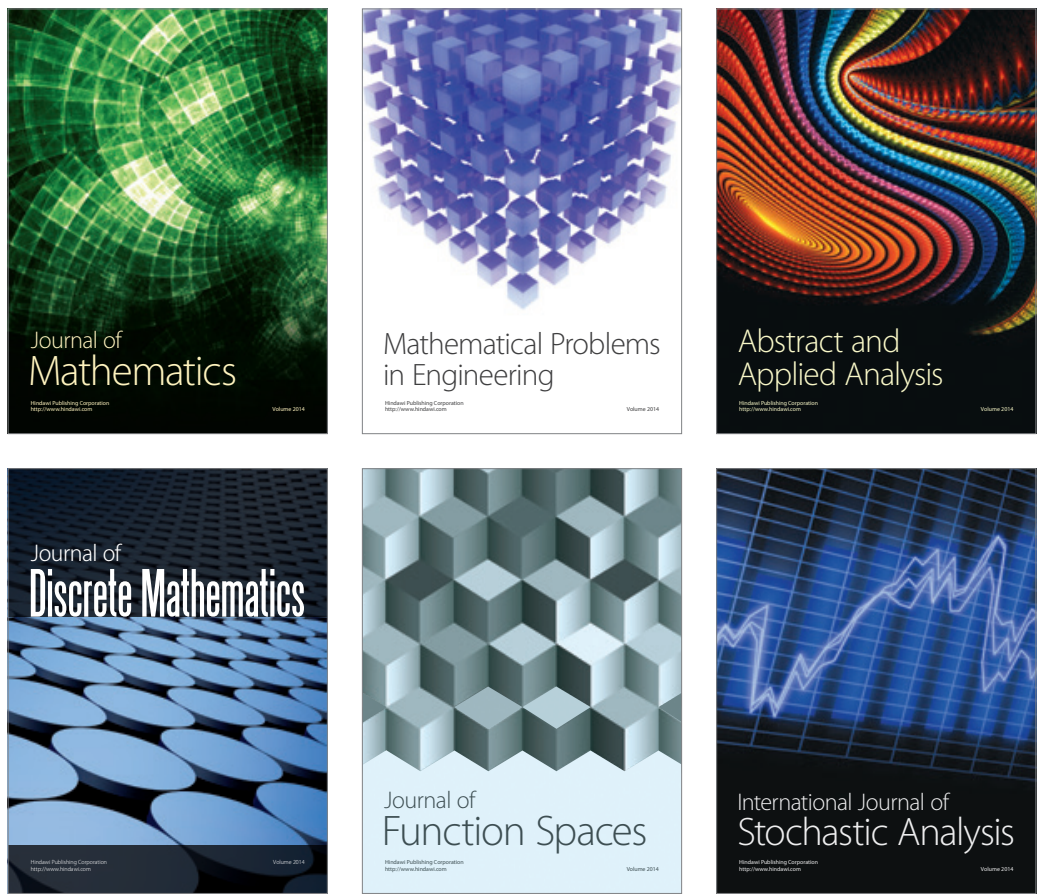

Journal of

Function Spaces

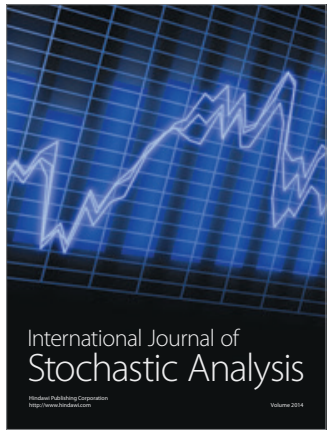

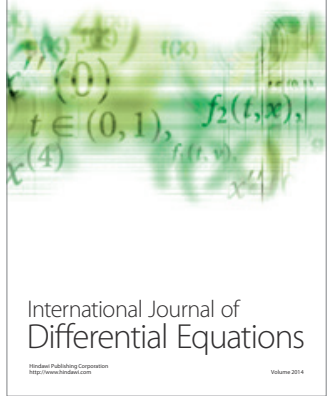
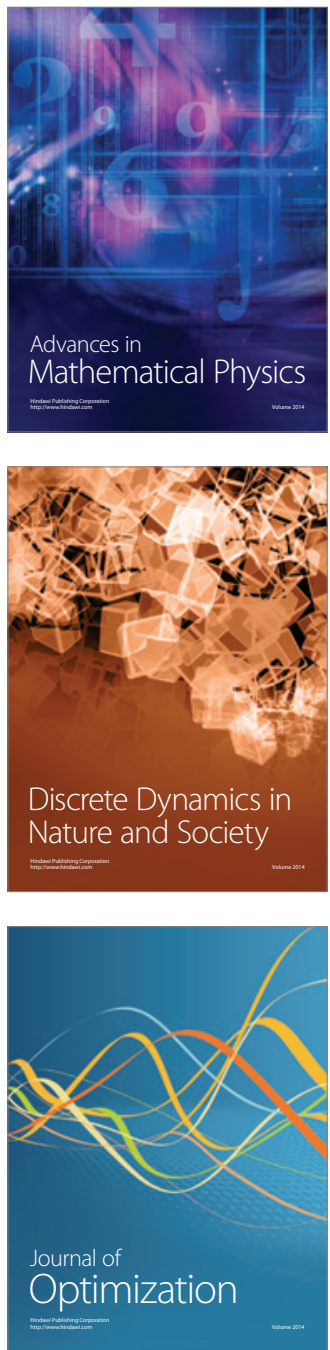